\title{
A New Form of Pseudo-elasticity in Small-scale Nanotwinned Gold
}

\author{
Chuang Deng ${ }^{1, *}$ and Frederic Sansoz ${ }^{2, *}$
}

\begin{abstract}
Molecular dynamics simulations are used to show a new type of pseudo-elasticity and shape memory effects in small-scale nanotwinned metals. Nanotwinned $\mathrm{Au}$ thin-films and nanowires are found to achieve full recovery of up to $20 \%$ tensile and $-6.25 \%$ compressive strains upon reverse loading when the twin boundaries make a special angle of $70.53^{\circ}$ from the principal axis. This phenomenon results in superelastic recoverable strains up to 5 times larger than the useful range of deformation that can be induced in some advanced bulk shape-memory-alloys and small-scale ceramics, with a tensile strength above $1 \mathrm{GPa}$. The pseudo-elastic behavior stems from a unique interplay between deformation twinning and slip in grains composed of non- $\left\{\begin{array}{lll}1 & 1 & 1\end{array}\right\}$ free surfaces and discontinued twin boundary migration in those exposing only $\left\{\begin{array}{lll}1 & 1 & 1\end{array}\right\}$ free surfaces. This finding could open up new opportunities for small-scale nanotwinned metals as advanced materials for vibration damping and mechanical energy storage applications.
\end{abstract}

Keywords: Pseudo-elasticity; shape memory effects; coherent twin boundary 


\section{Introduction}

Shape-memory effects and pseudo-elasticity have been widely observed in alloys that experience martensitic transformations subject to stress or temperature change[1-5]. With the growing interests in nanomaterials, new forms of phase transformation and shape-memory effects have been found in low-dimensional single-crystalline metals such as face-centered cubic (FCC) [6-9] and body-centered cubic (BCC) metal nanowires (NWs)[10,11] in recent years. As revealed by in-situ nanomechanical experiments[12] and atomistic simulations[6,9], pseudo-elasticity and shape memory effects were

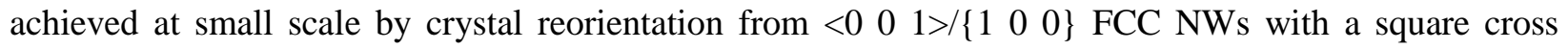

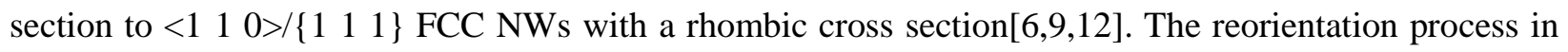
single crystal (SC) FCC NWs is mediated by deformation twinning and twin boundary (TB) migration[6,9,12], which differs from the martensitic transformation in conventional shape memory alloys[1-5].

In this letter, using molecular dynamics (MD) simulations, we report a different form of pseudo-elasticity and shape memory effects in small-scale metals containing nanotwins, with recoverable transformations involving neither crystal reorientation nor martensitic transformation. Nanotwinned metals have drawn considerable interest for their extreme tensile strength (over $1 \mathrm{GPa}$ ) and high ductility[13-16], compared to single-crystalline and nanocrystalline metals. However, past studies on the tension/compression of twinned metal NWs where pre-existing coherent twin boundaries (CTBs) were oriented either perpendicular or parallel to the NW axis and loading direction, did not find any evidence of pseudo-elasticity[15-18], although two recent studies reported recoverable plasticity in penta-twinned Ag NWs governed by dislocation nucleation and retraction[19,20]. Here, using Au thin-films and NWs as prototypes, we demonstrate that strong shape memory effects and pseudo-elasticity can prevail in nanotwinned metals if CTBs are oriented at a special angle of $19.47^{\circ}$ from the horizontal, i.e. a $70.53^{\circ}$ angle from the principal axis, which generally favors soft slip in the direction parallel to the twin plane. 


\section{Materials and Methods}

An Au film with tilted periodic nanotwins was constructed as shown in the inset of Figure 1. Since the theoretical angle between two neighbouring $\left\{\begin{array}{lll}1 & 1 & 1\end{array}\right\}$ planes in FCC metals is also $70.53^{\circ}$, the initially horizontal CTBs were rotated around the Y-axis $\left(<\begin{array}{lll}1 & 0\end{array}\right)$ ) by $19.47^{\circ}$ so that half of the free surface is turned into the $\left\{\begin{array}{lll}1 & 1 & 1\end{array}\right\}$ type, as indicated in Figure 1 . The dimensions of the simulation cell were $\sim 10 \mathrm{~nm}$ $\times 18 \mathrm{~nm} \times 18 \mathrm{~nm}$ along the $\mathrm{X}, \mathrm{Y}$, and $\mathrm{Z}$ directions as defined in Figure 1, and the spacing between CTBs was $\sim 4 \mathrm{~nm}$. Periodic boundary conditions were applied along the $\mathrm{Y}$ and $\mathrm{Z}$ directions, while the two surfaces perpendicular to the X-direction were set free. All simulations were performed using the MD simulation software LAMMPS[21] with an embedded atom method potential for $\mathrm{Au}[22]$. This potential was chosen for being able to reproduce consistent stacking fault energy and twinning fault energy for $\mathrm{Au}[16,17,23]$, which are key variables for predicting the dislocation nucleation and deformation twinning behavior in this metal under mechanical loading. The simulation time step was $5 \mathrm{fs}$ as in our previous atomistic studies of Au NW deformation[17,18,23]. The system was thermally relaxed at $300 \mathrm{~K}$ for 500 ps under zero pressure with isothermal-isobaric ensemble (NPT) prior to the uniaxial tensile or compressive deformation, which was performed at $300 \mathrm{~K}$ and a constant engineering strain rate of $5 \times$ $10^{8} \mathrm{~s}^{-1}$ with canonical ensemble (NVT) following the relaxation. The stress was calculated by adding the local Virial atomic stress along the loading direction over all atoms and dividing by the deformed volume[17,18,23]. AtomEye was used to visualize the atomistic configurations[24].

\section{Results}

The stress-strain curves for uniaxial tensile and compressive loadings are shown in Figure 1. Reverse loading imposed before reaching the maximum strength under both tension and compression are also represented, along with different deformed states marked by red circles on the stress-strain curves. Figure 1 shows that the loading-unloading behavior in the nanotwinned Au film is highly recoverable after the first loading cycle, albeit prior to the maximum tension and compression strengths, circles FC and ZT, 
respectively. A significant superelastic (pseudo-elastic) behavior extending up to $20 \%$ strain in tension and $-6.25 \%$ in compression is also evidenced. For comparison, we note that no pseudo-elasticity has been found in nanotwinned Au films of similar geometry but with perfectly horizontal $\left(0^{\circ}\right) \mathrm{CTBs}$. The total amount of superelastic recoverable deformation predicted in the Au thin-films with tilted CTBs ( 26\%) is 2-5 times larger than the useful range of deformation that can be induced in some advanced shape-memory-alloys (13\%)[25] and small-scale ceramics (8\%)[26] with a tensile strength above 1 GPa.

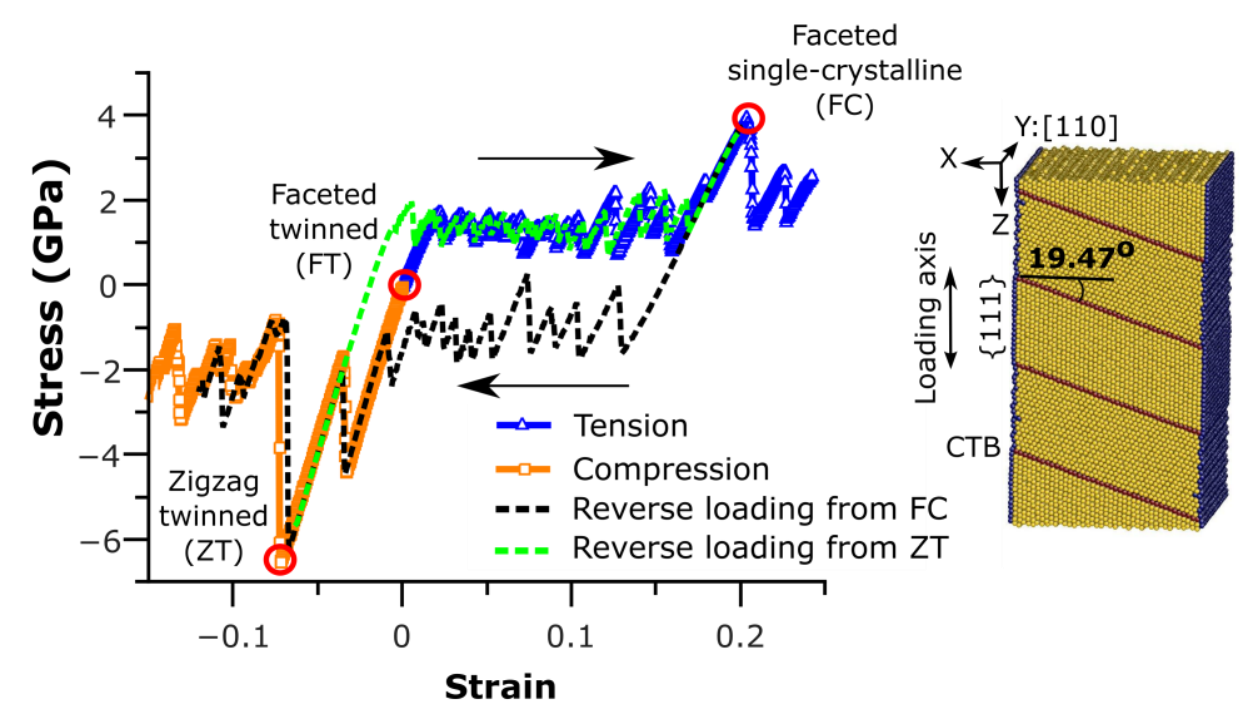

Figure 1. Stress-strain curves of a nanotwinned $\mathrm{Au}$ thin-film subjected to uniaxial tension and compression, or upon reverse loadings before reaching the maximum strengths in tension or compression. Representative deformation states are marked by red circles. The inset shows the atomistic model of the nanotwinned $\mathrm{Au}$ film with atoms colored according to their local lattice structure. Surface atoms are colored in blue, while CTBs and FCC atoms appear in red color and yellow color, respectively. The FT, FC, and ZT designations correspond to faceted twinned, faceted single-crystalline, and zigzag twinned deformations, respectively.

As shown in Figure 2, pseudo-elasticity in nanotwinned Au thin-films is accompanied by transformations of three distinct structures. Each of these structures, FT, FC and ZT in Figure 1, is very stable within a specific range of deformation. When the nanotwinned Au film is stress-free or loaded at a small strain 
under either tension or compression, the film maintained its original configuration, namely "flat-twinned" (FT) as shown in Figure 2. Upon tensile loading, the flat-twinned structure is gradually transformed into a defect-free "faceted" SC (FC) phase before reaching the maximum strength at $20 \%$ tensile strain, beyond which dislocations are nucleated and propagated in the crystal. In contrast, the flat-twinned structure in compression is transformed into a stable "zigzag-twinned" (ZT) structure at $-6.25 \%$ strain. Dislocation nucleation and sharp yielding is irreversibly caused if further compression strain is applied. As highlighted by circles in Figure 2(a), the zigzag-twinned structure consists of TBs surrounded by two $\{11$ 1) surface facets, which is different from TBs in the undeformed FT structure. This special ZT configuration has been previously reported in the literature, and was found to dramatically increase the strength of nanotwinned $\mathrm{Au}$ NWs[16,18,23]. In particular, MD simulations[16,18] and in-situ high-resolution transmission electron microscopy tensile tests [16,23] have shown evidence that ultrathin Au NWs with zigzag-twinned structure and nano-twins approach the ideal theoretical strength for this metal. Reverse loadings from $20 \%$ to $-6.25 \%$ strain and $-6.25 \%$ to $20 \%$ strain cyclically retransform the Au film between the ZT and FC structures, during which a FT structure is also recovered.

The intrinsic processes governing the transformation of the three distinct structures during reverse loading are detailed in Figure 2(a) and 2(b), respectively. Upon reverse loading from tension in Figure 1, Figure 2(a) shows that the Au film is changed from the FC structure at $20 \%$ strain to a twinned one at $7.7 \%$ strain through deformation twinning localized in the grains with non- $\{111\}$ free surfaces, see also Movie S1. The newly formed TBs make a perfect $19.47^{\circ}$ angle with respect to the loading axis, and migrate through shear coupling[27] to enlarge the TB spacing until the Au film is fully recovered to its original form, i.e., FT structure. Upon further compression, the TB migration continues towards the grains with only $\left\{\begin{array}{lll}1 & 1 & 1\end{array}\right\}$ free surfaces to form the zigzag-twinned structure at $-3.3 \%$ compression. Once transformed, the ZT configuration becomes very stable up to a compressive strain of $-6.25 \%$, indicative of discontinued TB migration. Without discontinued twin growth, the film would possibly return to a plastically-deformed 
detwinned structure with limited stability. Therefore discontinued TB migration plays an important role in the enhanced superelastic behavior predicted by simulation in Figure 1.

(a) $\quad$ FC

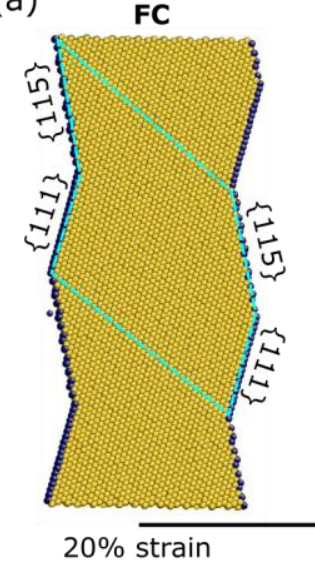

(b)

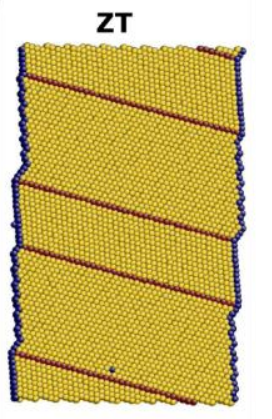

$-6.25 \%$ strain
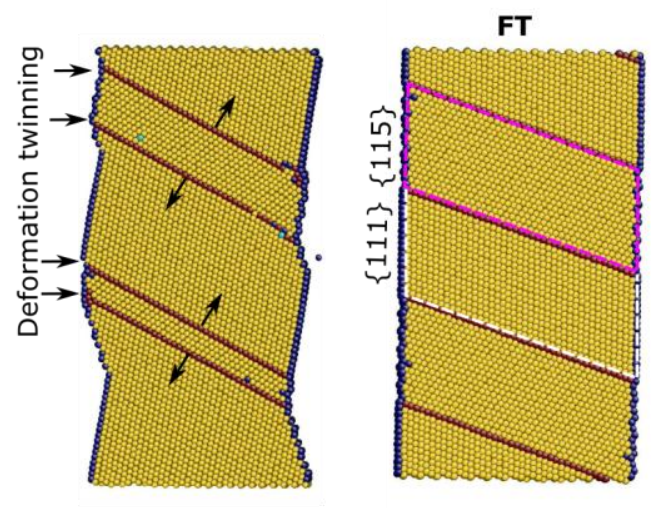

$0 \%$ strain
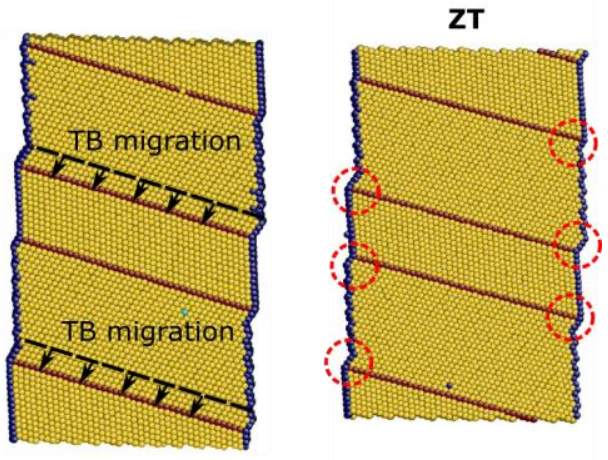

$-6.25 \%$ strain

FT

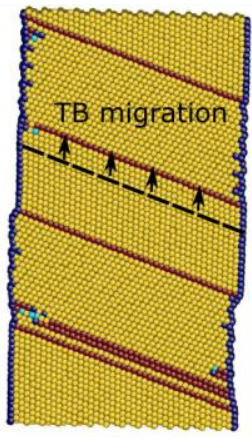

FC
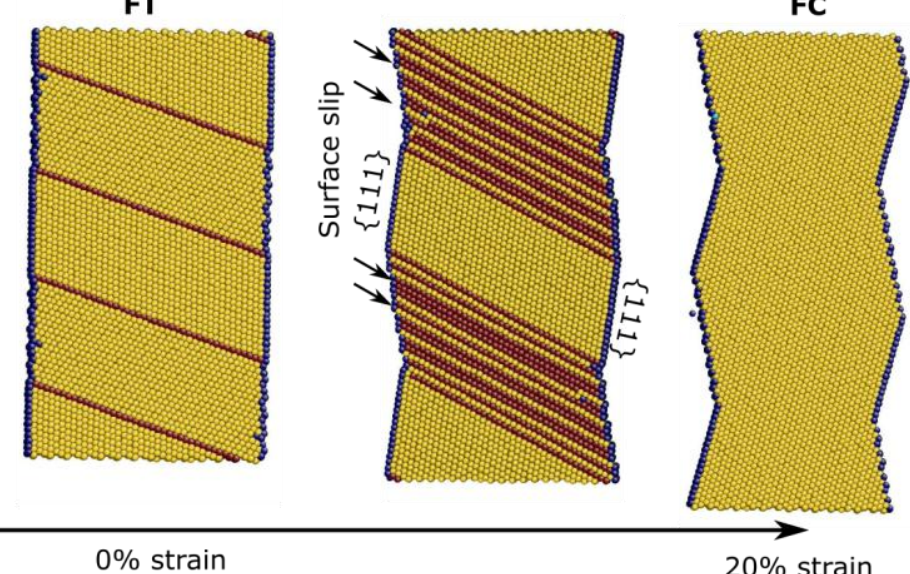

$0 \%$ strain

$20 \%$ strain

Figure 2. Atomistic configurations of the Au film at different deformation stages during reverse loading from (a) FC (20\% strain) and (b) ZT (-6.25\% strain), respectively. The atom color is assigned according to the local lattice structure. Red dashed circles are used to highlight the formation of special zigzag surface facets during compression.

The reverse transformation in compression from ZT at $-6.25 \%$ to FT Au films starts by detwinning of the $\{111\}$-faceted grains as shown in Figure 2(b) and movie S2. However, it is important to note that, since the direction of the resolved shear stress on the CTB planes is reversed between Figure 2(a) and Figure 
2(b), the TB migration occurs in opposite directions. Upon further tensile loading, the Au film completely returns to its original flat-twinned (FT) structure, followed by surface-mediated slip taking place in the grains with non- $\left\{\begin{array}{lll}1 & 1 & 1\end{array}\right\}$ type free surface. During this process, however, the newly formed $\left\{\begin{array}{lllll}1 & 1 & 1\end{array}\right\}$ slip planes continuously change sequence between the $\mathrm{ABC}$ stacking parallel to the $\mathrm{CTB}$ plane, thus forming hexagonal closed packed (HCP) stacking-faults in the grains with non- $\{111\}$ surfaces. The extended stacking-faults form an intermediate phase until the overall structure is converted back into a perfect FCC SC phase at $20 \%$ strain, Figure 2(b), to lower its overall energy; see also movie S2.

We emphasize that, in Figure 2(a), the CTBs can only migrate by surface nucleation of twinning partial dislocations in the grains with non- $\left\{\begin{array}{llll}1 & 1 & 1\end{array}\right\}$ free surface, but temporarily stop when a $\left\{\begin{array}{llll}1 & 1 & 1\end{array}\right\}$ surface facet is encountered. The TB migration is thus discontinued until a much higher compressive stress (the first peak during compressive loading) is reached to trigger the ZT configuration. Similarly surface slip only

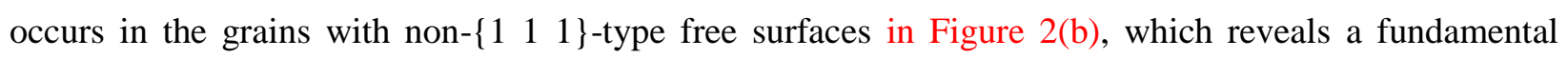
difference in surface dislocation nucleation processes between $\left\{\begin{array}{lll}1 & 1 & 1\end{array}\right\}$ and non- $\left\{\begin{array}{lll}1 & 1 & 1\end{array}\right\}$ free surfaces. It is therefore possible to assume that partial dislocation nucleation is a more difficult process in grains with $\left\{\begin{array}{lll}1 & 1 & 1\end{array}\right\}$ free surfaces than those with non- $\left\{\begin{array}{lll}1 & 1 & 1\end{array}\right\}$ free surfaces; for example, it has been reported by Park et al.[28] that no planar defects were observed in compression of FCC NWs with $\left\{\begin{array}{llll}1 & 1 & 1\end{array}\right\}$ side surfaces, while twinning and slip can be nucleated relatively easily in FCC NWs with other typical surface facet orientations such as $\left\{\begin{array}{lll}1 & 0 & 0\end{array}\right\}$ and $\left\{\begin{array}{lll}1 & 1 & 0\end{array}\right\}$.

To test this hypothesis, the critical resolved shear stresses required to initiate surface slip or TB migration from single crystals with either $\left\{\begin{array}{lll}1 & 1 & 1\end{array}\right\}$ or non- $\left\{\begin{array}{lll}1 & 1 & 1\end{array}\right\}$ surface facets were computed. On one hand, as shown in inset of Figure 3(a), SC models with either $\left\{\begin{array}{lll}1 & 1 & 1\end{array}\right\}$ or non- $\left\{\begin{array}{lll}1 & 1 & 1\end{array}\right\}$ free surfaces were constructed, based on the FT configuration outlined with pink and white dashed lines in Figure 2(a), to simulate the surface slip mechanism. Here the non- $\left\{\begin{array}{lll}1 & 1 & 1\end{array}\right\}$ facet is indexed to be $\left\{\begin{array}{lll}1 & 1 & \overline{5}\end{array}\right\}$. On the other hand, a SC model containing both $\left\{\begin{array}{llll}1 & 1 & 1\end{array}\right\}$ and $\left\{\begin{array}{llll}1 & 1 & \overline{5}\end{array}\right\}$ free surfaces, as shown in the inset of Figure 3(b), 
was made from the region outlined in green dashed lines in Figure 2(a), to simulate the TB migration mechanism. Simple shear deformation was applied to all models in the same way, by fixing a thin slab at the bottom of the SC Au film and moving another thin slab of atoms at the top at a constant velocity of 8 $\mathrm{m} / \mathrm{s}$ along the horizontal direction. In Figure 3(a), we have also considered shear deformation on two opposite directions as to simulate the slip behavior of Au films in Figure 2 under either compression or tension loading conditions. In Figure 3(b), the applied shear direction was identical to that produced by compression of the Au film in the FC configuration shown in Figure 2(a).

Figure 3(a) shows that the critical resolved shear stress to initiate surface slip at peak stress is lower in the model with $\left\{\begin{array}{lll}1 & 1 & \overline{5}\end{array}\right\}$ surface facets than that with $\left\{\begin{array}{lll}1 & 1 & 1\end{array}\right\}$ surface facets. The results also suggest that the necessary shear stress to initiate surface slip under compression is so high (2-3 GPa), compared to that in tension (0.8-1.2 GPa), that dislocation nucleation from the free surface is unlikely in compression. CTB migration is thus favored, consistent with the transformations shown in Figure 2(a).

The stress-strain curve in Figure 3(b) shows three stages of deformation for the TB migration mechanism. Stage I exhibits a sharp peak at which surface slip takes place at the top and bottom of the $\left\{\begin{array}{lll}1 & 1 & \overline{5}\end{array}\right\}$ faceted region, as shown in inset. Stage II is associated with a stick-slip behavior corresponding to the growth of twins by successive TB migration events due to shear coupling effects[27]. As shown in inset, twin growth is also limited to the region with $\left\{\begin{array}{lll}1 & 1 & \overline{5}\end{array}\right\}$ surface facets. Once the top and bottom twins meet and annihilate each other in the middle, the CTB at the bottom starts to migrate downwards in the region with $\left\{\begin{array}{lll}1 & 1 & 1\end{array}\right\}$-surface facets, leading to Stage III. It is clearly shown by the stress-strain curve in Figure 3(b) that the stress required for CTB migration is indeed significantly larger in the region with $\left\{\begin{array}{llll}1 & 1 & 1\end{array}\right\}$ surface facets (Stage III) than in that with $\left\{\begin{array}{lll}1 & 1 & \overline{5}\end{array}\right\}$ surface facets (Stage II). Movie S3 shows the details of the entire process described in Figure 3(b). 

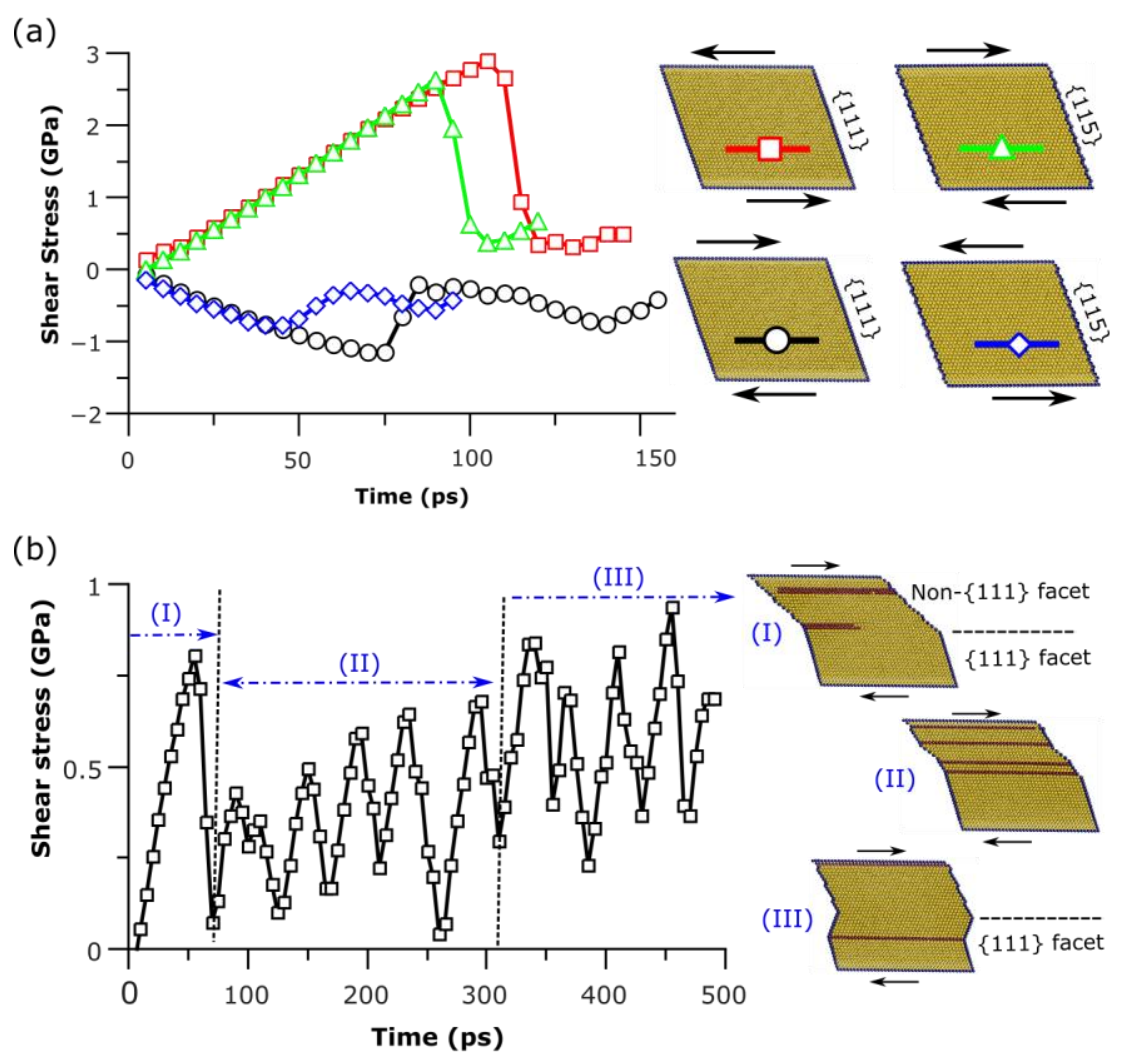

Figure 3. Atomistic simulations of simple shear deformation in SC Au films with (a) either $\left\{\begin{array}{lll}1 & 1 & \overline{5}\end{array}\right\}$ or $\{1$ $11\}$ surface facets to simulate surface slip mechanisms, and (b) both $\left\{\begin{array}{lll}1 & 1 & \overline{5}\end{array}\right\}$ and $\left\{\begin{array}{lll}1 & 1 & 1\end{array}\right\}$ surface facets to simulate deformation twinning and shear-coupled TB migration mechanisms. The inset shows the corresponding atomistic configurations with atom color assigned according to the local lattice structure.

Since CTBs are initially present in the un-deformed Au films, and are involved during most of the transformations shown in Figure 2, it is important to clarify that the pseudo-elasticity and transformation mechanism predicted here are different from shape-memory effects and pseudo-elastic behaviors reported previously in FCC NWs, i.e. through purely deformation twinning and TB migration. Although deformation twinning and TB migration was nevertheless important for the transformation from $\mathrm{FC}$ to $\mathrm{ZT}$ and ZT to FC configurations as shown in Figure 2(a) and 2(b) respectively, no crystal re-orientation between $\left\langle\begin{array}{lll}1 & 0 & 0\end{array}\right\rangle$ and $\left\langle\begin{array}{lll}1 & 1 & 0\end{array}\right\rangle$ was observed as previously reported in FCC metal NWs showing pseudo-elasticity. On the contrary, neither the original nor the final transformed Au films are oriented 
along $\langle 100\rangle$ or $\langle 110>$ directions during the whole process. Furthermore, for tension between $0.5 \%$ to $7.6 \%$ strain, the transformation from the original FT to FC structures is achieved though continuous surface slip; here deformation twinning and TB migration play little role, if any, during this stage of deformation.

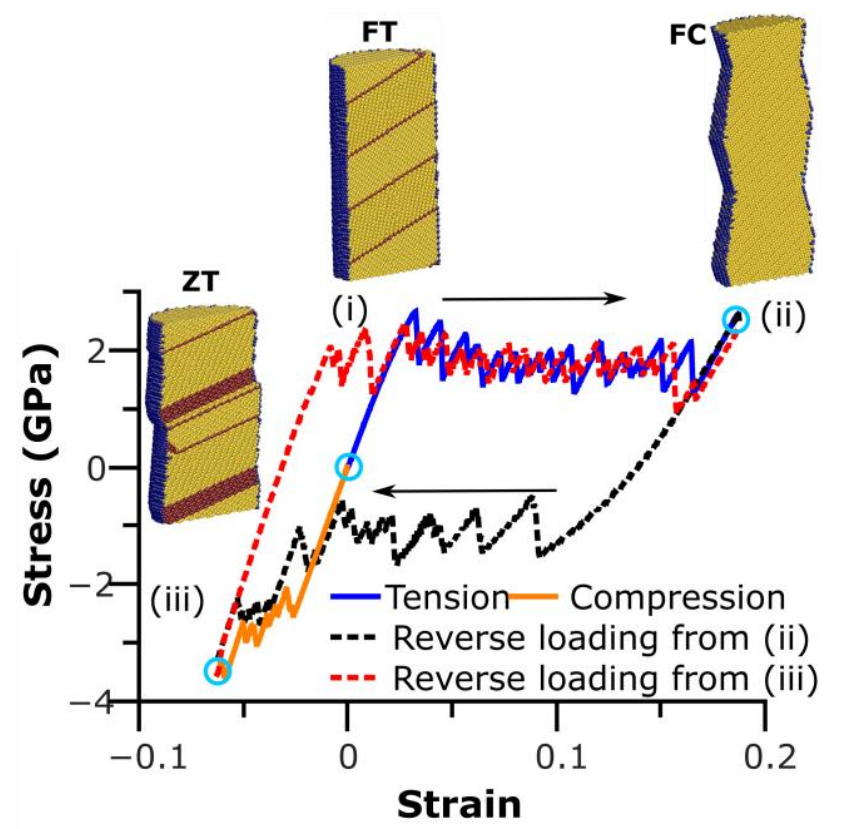

Figure 4. Stress-strain curves of a nanotwinned $\mathrm{Au}$ NW during tension or compression, and the respective reverse loadings before the maximum strength. Representative states of deformation (i)-(iii) were marked by blue circles. The inset shows the atomistic model of the nanotwinned Au NW with atoms colored according to their local lattice structure. The Au NW front has been removed to show the interior structure.

So far, it has been demonstrated that pseudo-elasticity can be achieved in the model system of nanotwinned $\mathrm{Au}$ film with the CTBs oriented at a special angle. However, a similar mechanism and pseudo-elasticity could be expected in other types of small-scale FCC metals. For example, pseudo-elasticity based on the re-orientation between $\left\langle\begin{array}{llll}0 & 0\end{array}\right\rangle$ and $\langle 111\rangle$ NWs has been widely reported in various types of FCC NWs including $\mathrm{Au}[12], \mathrm{Cu}[9]$ and $\mathrm{Ag}[7]$. Therefore it is important to test if the 
concept can be extended to other low-dimensional materials such as metal NWs, which are of practical importance for nanoelectromechanical systems. For that purpose, a cylindrical nanotwinned Au NW with a diameter of $10 \mathrm{~nm}$ was cut from the nanotwinned Au film model, as shown in state (i) in the inset of Figure 4. The front of the Au NW is removed in this figure to reveal the interior structure. Tensile and compressive deformation in the Au NW was simulated under the same conditions than the nanotwinned Au films. The stress-strain curves and associated deformation snapshots shown in Figure 4 confirm that the pseudo-elasticity observed in nanotwinned Au films is also present in the nanotwinned Au NW: Full recovery between three distinct structures, FT, FC and ZT Au NWs, was evidenced upon loading and reverse loading, between $-6.25 \%$ and $18.75 \%$ strains.

We note that pseudo-elasticity and shape memory effects in FCC NWs based on crystal re-orientation between $\left\langle 1 \quad 0 \quad 0>\right.$ and $\left\langle\begin{array}{lll}1 & 1 & 0\end{array}>\right.$ were found to be driven by temperature effects [6], whereas pseudo-elasticity and structural transformations shown in Figure 4 only required stress-driven surface slip. To confirm the lack of temperature dependence in our simulations, we have performed additional simulations at high temperatures from $800 \mathrm{~K}$ to $1200 \mathrm{~K}$ without mechanical deformation, which showed no NW transformations.

Also it is worth mentioning that a temporary FCC-to-HCP phase transformation was observed during surface slip as shown in inset of Figure 4. The formation of HCP phase in Au has been reported in past experimental and MD simulation studies[29],30]. However, the HCP phase in this figure was found to not be stable, transforming back to FCC phase via sequential surface slip along $\left\{\begin{array}{llll}1 & 1 & 1\end{array}\right\}$ planes.

\section{Discussion}

It needs to be emphasized that the pseudo-elasticity in nanotwinned cylindrical metal NWs largely depends on the TB orientation, the TB spacing, and the aspect ratio of the NW. For example, no pseudo-elasticity and transformations between the three configurations was found in nanotwinned $\mathrm{Au}$ 
NWs when the initially horizontal CTBs were rotated around the $\langle 112\rangle$ (x-axis) direction, instead of the $<110>$ (y-axis) direction. It was also found that rotating the CTBs around $<110>$ (y-axis) direction by an angle slightly different from $19.47^{\circ}$ still resulted in pseudo-elasticity, but the recovery strain was not as high as when the rotation angle was $19.47^{\circ}$. Furthermore, the deformation of cylindrical Au NWs with TBs oriented in the same way than those in Figure 4, but with a larger diameter of $20 \mathrm{~nm}$ (length of $54 \mathrm{~nm}$ ) and two different TB spacings ( $4 \mathrm{~nm}$ and $8 \mathrm{~nm}$ ) were simulated under the same loading conditions. It was found that the transformation from the original FT configuration to ZT structure upon monotonic compression or FC structure upon monotonic tension was possible in both Au NWs, but the full recovery between the FT and FC structures was only achieved in the Au NW with TB spacing $=8 \mathrm{~nm}$. These results therefore suggest that a combination of relatively small aspect ratio and large spacing between the CTBs is desirable to improve the recovery and pseudo-elasticity in nanotwinned Au NWs. The main reason is that NWs with a high aspect ratio will experience severe buckling during compression, which prohibits a full recovery of the NWs during reverse loading. Furthermore, a relatively large TB spacing is important to allow sufficient space for successive surface sip and CTB migration, which are important for the recovery process. When the TB spacing is small, the transformed FC structure contains more facets. Therefore, during reverse loading, surface slip and deformation twinning are more easily localized on the surface facets, resulting in buckling and early failure of the NW before the full recovery is achieved. This may be the reason why pseudo-elasticity was not observed in the work by Jang et al.[15] who performed in-situ nanomechanical tension test of twinned $\mathrm{Cu}$ nanopillars with the $\mathrm{CTB}$ silted by $18^{\circ}$; the TB spacing in their samples was $0.63 \mathrm{~nm} \sim 1.25 \mathrm{~nm}$. Although another experimental study has investigated the effect of twin orientation on mechanical properties in metals[31], the new form of pseudo-elasticity found in this work has not yet been reported in the literature. However, it is expected that proof of concept experiments based on this study can be designed through in-situ tension and compression[15,32-34] of nanotwinned metal pillars by carefully controlling the TB spacing, the aspect ratio, and TB orientations. 


\section{Conclusions}

We have found a new form of pseudo-elasticity in nanotwinned Au films and Au NWs with the TBs oriented at a special angle by using MD simulations. Transformation of three recoverable structures was predicted in nanotwinned Au thin-films and NWs upon tensile and compressive loadings, over a wide range of deformation up to $26 \%$ strain $(20 \%$ tension and $-6.25 \%$ compression). The observed superelasticity is larger than that of advanced bulk shape-memory-alloys and small-scale ceramics with tensile strengths above $1 \mathrm{GPa}$. The pseudo-elastic transformations in small-scale nanotwinned metals were found to result from facet-dependent surface slip and discontinued TB migration processes, which are different from the pseudo-elastic behavior and shape memory effects in FCC metal NWs that have been reported before. The conclusions of this study are also broadly important for understanding the role of nanotwins in small-scale materials for vibration damping and mechanical storage energy applications.

\section{Acknowledgments}

This work was supported by NSERC Discovery Grant under RGPIN 430800-2013, Canada, and enabled by the use of computing resources provided by WestGrid and Compute/Calcul Canada. FS also thanks support from NSF grant DMR-1410646.

\section{References}

[1] K. Otsuka, C.M. Wayman, Shape memory materials, Cambridge university press, 1999.

[2] K. Otsuka, X. Ren, Physical metallurgy of Ti-Ni-based shape memory alloys, Prog. Mater. Sci. 50 (2005) 511-678.

[3] H.Y. Kim, Y. Ikehara, J.I. Kim, H. Hosoda, S. Miyazaki, Martensitic transformation, shape memory effect and superelasticity of $\mathrm{Ti}-\mathrm{Nb}$ binary alloys, Acta Mater. 54 (2006) 2419-2429.

[4] J. San Juan, M.L. Nó, C.A. Schuh, Nanoscale shape-memory alloys for ultrahigh mechanical damping, Nat. Nanotechnol. 4 (2009) 415-419.

[5] M. Wuttig, J. Li, C. Craciunescu, A new ferromagnetic shape memory alloy system, Scr. Mater. 44 (2001) 2393-2397.

[6] H.S. Park, K. Gall, J.A. Zimmerman, Shape memory and pseudoelasticity in metal nanowires, Phys. Rev. Lett. 95 (2005) 255504. 
[7] H.S. Park, C. Ji, On the thermomechanical deformation of silver shape memory nanowires, Acta Mater. 54 (2006) 2645-2654.

[8] W. Liang, M. Zhou, Pseudoelasticity of single crystalline $\mathrm{Cu}$ nanowires through reversible lattice reorientations, J. Eng. Mater. Technol. 127 (2005) 423-433.

[9] W. Liang, M. Zhou, F. Ke, Shape memory effect in Cu nanowires, Nano Lett. 5 (2005) 2039-2043.

[10] A. Cao, Shape memory effects and pseudoelasticity in bcc metallic nanowires, J. Appl. Phys. 108 (2010) 113531.

[11] L. Sandoval, H.M. Urbassek, Finite-Size Effects in Fe-Nanowire Solid- Solid Phase Transitions: A Molecular Dynamics Approach, Nano Lett. 9 (2009) 2290-2294.

[12] J.-H. Seo, Y. Yoo, N.-Y. Park, S.-W. Yoon, H. Lee, S. Han, et al., Superplastic deformation of defect-free $\mathrm{Au}$ nanowires via coherent twin propagation, Nano Lett. 11 (2011) 3499-3502.

[13] L. Lu, Y. Shen, X. Chen, L. Qian, K. Lu, Ultrahigh strength and high electrical conductivity in copper, Science. 304 (2004) 422-426.

[14] X. Zhang, O. Anderoglu, R.G. Hoagland, A. Misra, Nanoscale growth twins in sputtered metal films, Jom. 60 (2008) 75-78.

[15] D. Jang, X. Li, H. Gao, J.R. Greer, Deformation mechanisms in nanotwinned metal nanopillars, Nat. Nanotechnol. 7 (2012) 594-601.

[16] J. Wang, F. Sansoz, J. Huang, Y. Liu, S. Sun, Z. Zhang, et al., Near-ideal theoretical strength in gold nanowires containing angstrom scale twins, Nat. Commun. 4 (2013) 1742.

[17] C. Deng, F. Sansoz, Enabling Ultrahigh Plastic Flow and Work Hardening in Twinned Gold Nanowires, Nano Lett. 9 (2009) 1517-1522.

[18] C. Deng, F. Sansoz, Near-Ideal strength in gold nanowires achieved through microstructural design, ACS Nano. 3 (2009) 3001-3008.

[19] R.A. Bernal, A. Aghaei, S. Lee, S. Ryu, K. Sohn, J. Huang, et al., Intrinsic Bauschinger effect and recoverable plasticity in pentatwinned silver nanowires tested in tension, Nano Lett. 15 (2014) 139-146.

[20] Q. Qin, S. Yin, G. Cheng, X. Li, T.-H. Chang, G. Richter, et al., Recoverable plasticity in penta-twinned metallic nanowires governed by dislocation nucleation and retraction, Nat. Commun. 6 (2015) 5983.

[21] S. Plimpton, Fast Parallel Algorithms for Short-Range Molecular Dynamics, J. Comput. Phys. 117 (1995) 1-19.

[22] G. Grochola, S.P. Russo, I.K. Snook, On fitting a gold embedded atom method potential using the force matching method, J. Chem. Phys. 123 (2005) 204719.

[23] J. Wang, F. Sansoz, C. Deng, G. Xu, G. Han, S.X. Mao, Strong Hall-Petch Type Behavior in the Elastic Strain Limit of Nanotwinned Gold Nanowires, Nano Lett. 15 (2015) 3865-3870.

[24] J. Li, AtomEye: an efficient atomistic configuration viewer, Model. Simul. Mater. Sci. Eng. 11 (2003) 173.

[25] Y. Tanaka, Y. Himuro, R. Kainuma, Y. Sutou, T. Omori, K. Ishida, Ferrous polycrystalline shape-memory alloy showing huge superelasticity, Science. 327 (2010) 1488-1490.

[26] A. Lai, Z. Du, C.L. Gan, C.A. Schuh, Shape memory and superelastic ceramics at small scales, Science. 341 (2013) 1505-1508.

[27] Q. Hu, L. Li, N.M. Ghoniem, Stick-slip dynamics of coherent twin boundaries in copper, Acta Mater. 57 (2009) 4866-4873. 
[28] H.S. Park, K. Gall, J.A. Zimmerman, Deformation of FCC nanowires by twinning and slip, J. Mech. Phys. Solids. 54 (2006) 1862-1881.

[29] Z. Fan, X. Huang, Y. Han, M. Bosman, Q. Wang, Y. Zhu, et al., Surface modification-induced phase transformation of hexagonal close-packed gold square sheets, Nat. Commun. 6 (2015) 6571.

[30] J. Diao, K. Gall, M.L. Dunn, Surface stress driven reorientation of gold nanowires, Phys. Rev. B. 70 (2004) 075413.

[31] A. Kobler, A.M. Hodge, H. Hahn, C. Kübel, Orientation dependent fracture behavior of nanotwinned copper, Appl. Phys. Lett. 106 (2015) 261902.

[32] D. Jang, J.R. Greer, Transition from a strong-yet-brittle to a stronger-and-ductile state by size reduction of metallic glasses, Nat. Mater. 9 (2010) 215-219.

[33] M. Legros, D.S. Gianola, C. Motz, Quantitative in situ mechanical testing in electron microscopes, MRS Bull. 35 (2010) 354-360.

[34] G. Richter, K. Hillerich, D.S. Gianola, R. Monig, O. Kraft, C.A. Volkert, Ultrahigh strength single crystalline nanowhiskers grown by physical vapor deposition, Nano Lett. 9 (2009) 3048-3052. 